\title{
Classification of quantum phases for the star-lattice antiferromagnet via a projective symmetry group analysis
}

\author{
Ting-Pong Choy and Yong Baek Kim \\ Department of Physics, University of Toronto, Toronto, Ontario, Canada M5S 1A7 \\ (Received 21 April 2009; revised manuscript received 21 July 2009; published 13 August 2009)
}

\begin{abstract}
We study possible quantum ground states of the Heisenberg antiferromagnet on the star lattice, which may be realized in the recently discovered polymeric iron acetate, $\mathrm{Fe}_{3}\left(\mu_{3}-\mathrm{O}\right)(\mu-\mathrm{OAc})_{6}\left(\mathrm{H}_{2} \mathrm{O}\right)_{3}\left[\mathrm{Fe}_{3}\left(\mu_{3}-\mathrm{O}\right)(\mu-\mathrm{OAc})_{7.5}\right]_{2} \cdot 7 \mathrm{H}_{2} \mathrm{O}$ [Y. Z. Zheng et al., Angew. Chem. Int. Ed. 46, 6076 (2007)]. Even though the $\mathrm{Fe}^{\mathrm{III}}$ moment in this material carries spin-5/2 and the system eventually orders magnetically at low temperatures, the magnetic ordering temperature is much lower than the estimated CurieWeiss temperature, revealing the frustrated nature of the spin interactions. Anticipating that a lower spin analog of this material may be synthesized in future, we investigate the effect of quantum fluctuations on the starlattice antiferromagnet using a large- $N \mathrm{Sp}(N)$ mean field theory and a projective symmetry group analysis for possible bosonic quantum spin liquid phases. It is found that there exist only two distinct gapped $Z_{2}$ spin liquid phases with bosonic spinons for nonvanishing nearest-neighbor valence-bond amplitudes. In particular, the spin liquid phase which has a lower energy in the nearest-neighbor exchange model can be stabilized for relatively higher spin magnitudes. Hence, it is perhaps a better candidate for the realization of quantum spin liquid state. We also determine the magnetic ordering patterns resulting from the condensation of the bosonic spinons in the two different spin liquid phases. We expect these magnetic ordering patterns would directly be relevant for the low temperature ordered phase of the iron acetate. The phase diagram containing all of these phases and various dimerized states are obtained for the nearest-neighbor exchange model and its implications are discussed.
\end{abstract}

DOI: 10.1103/PhysRevB.80.064404

PACS number(s): 75.10.Jm, 71.10.Hf

\section{INTRODUCTION}

The search for quantum spin liquid phases in two and three dimensions has lead to recent discoveries of several spin-1/2 frustrated antiferromagnets, where no magnetic ordering has been seen down to the lowest temperature. The examples include a triangular lattice organic material close to a metal-insulator transition, ${ }^{2}$ Kagome or Kagome-like lattice systems, ${ }^{3-5}$ and a three-dimensional hyber-Kagome lattice material. ${ }^{6}$ The nature of possible spin liquid and other competing phases in these systems has been a subject of intense research activities. While there has been considerable progress in understanding some of the candidate quantum paramagnetic phases such as quantum spin liquid ${ }^{7-13}$ and valence bond solid phases, ${ }^{14-16}$ a general understanding of the interplay between competing phases upon the variation of the spin interactions is still lacking. ${ }^{17}$ Therefore, systematic studies of a variety of frustrated magnets with possibly different spin interactions and/or with different underlying lattice structures would be extremely useful. ${ }^{18,19}$

In this regard, the recent discovery of the iron acetate may present one of such useful examples for a two-dimensional frustrated lattice. ${ }^{1}$ Here, $\mathrm{Fe}^{\mathrm{III}}$ spin-5/2 moments reside on the star lattice as shown in Fig. 1. The Curie-Weiss temperature is estimated to be $\Theta_{\mathrm{CW}}=-581 \mathrm{~K}$, but the magnetic ordering occurs only below $T_{N}=4.5 \mathrm{~K}$ (the nature of the magnetic order is presently not known), leading to a large frustration parameter, $f=\left|\Theta_{\mathrm{CW}}\right| / T_{N}=129 \gg 1$. This raises the hope that spin liquid phases may exist for a lower spin analog of this material.

In this paper, we investigate possible quantum ground states of the star-lattice antiferromagnet, including quantum spin liquid phases, magnetically ordered states, and dimerized phases using a projective symmetry group analysis ${ }^{20}$ and a large- $N \operatorname{Sp}(N)$ mean-field theory. ${ }^{21,22}$ We expect the quantum paramagnetic phases, namely the spin liquid and dimerized phases, may be relevant to a lower spin analog (e.g., spin-1/2 or spin-1) of the iron acetate, which is yet to be discovered. The magnetically ordered phases described in this work may directly be relevant to the low temperature ordered phase of the iron acetate.

The star lattice can be regarded as a triangular Bravais lattice with a six-site basis and hence the unit cell contains

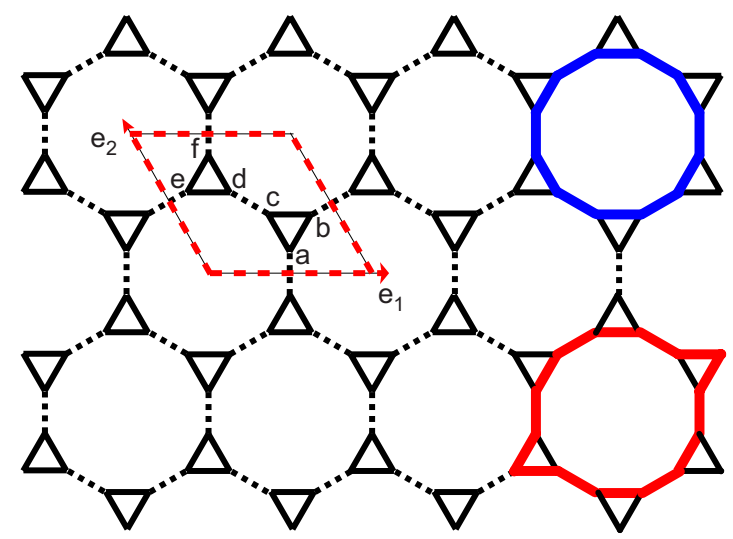

FIG. 1. (Color online) Star lattice is shown with two inequivalent nearest neighbor spin exchange interactions $J_{t}$ and $J_{d}$ along the triangular and bridge links, denoted by the solid and dotted lines, respectively. The rhombus enclosed by dashed lines corresponds to a unit cell with six sites labeled by the indices $a$ to $f$. Here, the 12-sided and 14-sided loops are also shown. 
six lattice sites as shown in Fig. 1. One can also view this lattice as a variant of the Kagome lattice in the sense that additional lattice links between triangles of the Kagome lattice are introduced. This leads to two topologically inequivalent nearest-neighbor spin exchange interactions: $J_{t}$ along the triangular links and $J_{d}$ along the bridge links that connect triangles. In the Heisenberg model with the antiferromagnetic sign for both $J_{d}$ and $J_{t}\left(J_{t}>0, J_{d}>0\right)$, there is clearly a macroscopic degeneracy of the classical ground states. ${ }^{23}$ Previous exact diagonalization studies of the spin-1/2 nearest-neighbor antiferromagnetic Heisenberg model on the star lattice suggest that the ground state may be a dimerized state with dimers sitting on the bridge links for $J_{t}=J_{d}$ and a threefold degenerate valence bond solid state when $J_{t}>1.3 J_{d} \cdot{ }^{23,24}$ The finite size effect in these studies, however, makes it difficult to draw a definite conclusion. Various models including the quantum dimer model ${ }^{25}$ and Kiteav model ${ }^{26}$ on the star lattice have also been studied recently.

In this work, we provide systematic understanding of possible quantum spin liquid phases with bosonic spinons in the star-lattice antiferromagnet using a projective symmetry group analysis of the mean-field states in the Schwinger boson theory. The projective symmetry group is a powerful tool to classify all and only the physical spin liquid states without specifying a particular spin Hamiltonian. We also investigate how these spin liquid states may be related to the previouslyidentified dimerized phases ${ }^{23,24}$ in the global phase diagram using a large- $N \mathrm{Sp}(N)$ Schwinger boson mean field theory.

Here we focus on the $Z_{2}$ spin liquid phases on the star lattice, where $Z_{2}$ represents a global pure gauge degree of freedom that leaves the mean-field states invariant. It has been shown that such $Z_{2}$ spin liquid states naturally arise in the Schwinger boson theory of the antiferromagnetic Heisenberg model on frustrated lattices. ${ }^{21}$ The projective symmetry group analysis leads to only a finite number of such $Z_{2}$ spin liquid phases. If we further require the system to have only nontrivial nearest-neighbor valence bond amplitudes, there exist only two distinct $Z_{2}$ (symmetric) spin liquid phases that preserve all the space group, spin rotation, and time reversal symmetries in contrast to the four symmetric $Z_{2}$ spin liquid states on the Kagome lattice. ${ }^{22}$ These two states can also be distinguished by the "flux" enclosed in the 12-sided loop as shown in Fig. 1, which is defined as the phase of the gaugeinvariant product of the valence-bond-amplitudes $Q_{i j}$ along the 12-sided loop, i.e., $\Theta=\arg \left[Q_{1,2}\left(-Q_{2,3}^{*}\right) Q_{3,4} \cdots\left(-Q_{12,1}^{*}\right)\right] .{ }^{27}$ The two $Z_{2}$ spin liquid phases correspond to $\Theta=0$ and $\Theta$ $=\pi$, respectively. The zero-flux state is an analog of the $[0 \mathrm{Hex}, \pi \mathrm{Rhom}]$ phase of the Kagome lattice. ${ }^{22}$ We study the spinon and spin-1 excitation spectra in the two spin liquid phases. In principle, the spin-1 excitation spectra can be measured by neutron scattering experiment to distinguish these two phases when an ambiguity as to the nature of the underlying quantum paramagnetic phase arises.

Using the results above, we also investigate possible magnetically ordered phases via the condensation of bosonic spinons in each spin liquid phase. The magnetic order arising from the zero-flux state has the magnetic ordering wavevector $\mathbf{q}= \pm\left(\frac{\pi}{3}+n \pi, \frac{\pi}{3}+m \pi\right)$ and $(n \pi, m \pi)$ with integers $n, m$. On the other hand, the magnetic ordering arising from the $\pi$-flux state has the ordering wavevector $\mathbf{q}=(0,0)$ and does not break translational symmetry. These results may directly be relevant to the low temperature magnetically ordered state of the iron acetate. The determination of the magnetic ordering wavevector would also tell us which spin liquid phase may close by. ${ }^{28}$

The relative stability of all these phases and the previously studied dimerized states ${ }^{23,24}$ is studied in a large- $N$ $\operatorname{Sp}(N)$ mean field theory of the nearest-neighbor exchange model $^{21,22}$ and the global phase diagram is obtained as a function of the effective spin magnitude $\kappa=2 S_{\text {eff }}$ and $J_{t} / J_{d}$. The advantage of the large- $N \operatorname{Sp}(N)$ theory is that one can treat the magnetically ordered and paramagnetic states on equal footing and the method is nonperturbative in the effective spin magnitude, $\kappa=2 S_{\text {eff }}$. The results are shown in Fig. 6 . It is found that the zero-flux state is always energetically favorable over the $\pi$-flux state in the nearest-neighbor model. In contrast to Kagome lattice, the critical $\kappa$ beyond which a magnetic order sets in, is much larger for the zeroflux state, i.e., $\kappa_{c}$ of the zero-flux phase can be as large as $\kappa_{c} \sim 5$ while the largest $\kappa_{c} \sim 1.5$ for the $\pi$-flux state. $\kappa_{c} \sim 5$ is an unusually large number because $\kappa_{c}$ is often smaller than unity in many cases. ${ }^{22}$ In fact, this is even larger than $\kappa_{c}$ $\sim 2$ of the $[0 \mathrm{Hex}, \pi \mathrm{Rhom}]$ phase of the Kagome lattice. This suggests that the zero-flux phase may exist even for relatively large spin $(S>1 / 2)$ system in an anisotropic limit.

In the ultimate quantum limit, $\kappa \ll 1$, the dimerized state with the dimers sitting on the $J_{d}$ bonds becomes the ground state when $J_{d}>J_{t}$ while only the spin correlations on the triangles survives in the opposite limit, $J_{d}<J_{t}$ (for the nearest-neighbor model). ${ }^{27}$ The dimerized state for $J_{d}>J_{t}$ is consistent with the previous numerical result ${ }^{23,24}$ on the spin$1 / 2$ nearest-neighbor Heisenberg model. The nature of the dimerized state for $J_{d}<J_{t}$ cannot clearly be identified in the present work because it requires further analysis of the $1 / N$ fluctuation corrections. ${ }^{29,30} \mathrm{We}$ emphasize, however, that the phase boundaries of various phases may look different in the physical $N=1$ limit, so the phase diagram obtained in the large- $N$ limit should be taken with a grain of salt. Further, it is possible that the inclusion of other spin interactions may favor the spin liquid over the dimerized states even deep inside the quantum regime $\kappa \ll 1$. The nature of the transitions between various phases in the phase diagram is also discussed in the main text of the paper.

The rest of the paper is organized as follows. In Sec. II, we briefly review an $\operatorname{Sp}(N)$ mean field theory of the antiferromagnetic Heisenberg model. In Sec. III, the concept of projective symmetry group (PSG) is introduced. Here, the PSG on the star lattice is applied to the $\operatorname{Sp}(N)$ mean field theory and is used to analyze possible $Z_{2}$ spin liquid phases. In Sec. IV, various physical properties of two distinct $Z_{2}$ spin liquid phases are explained and the mean-field phase diagram including dimerized and magnetically ordered phases (for the nearest-neighbor Heisenberg model) is obtained. We discuss the implications of our results to theory and experiment in Sec. V. Details of the derivation of the PSG for the star lattice are given in Appendix.

\section{SP( $N)$ GENERALIZATION OF THE HEISENBERG MODEL}

To investigate possible magnetically ordered and quantum paramagnetic states in the quantum antiferromagnetic 
Heisenberg model, $H=\sum_{i j} J_{i j} \mathbf{S}_{i} \cdot \mathbf{S}_{j}$, it is useful to generalize the usual spin-SU(2) Heisenberg model to an $\operatorname{Sp}(N)$ model. 21,33

Let us start with the Schwinger boson representation of the spin operator $\mathbf{S}_{i}=b_{i \alpha}^{\dagger} \sigma_{\alpha \beta} b_{i \beta}$, where $\alpha, \beta=\uparrow, \downarrow, \sigma$ are Pauli matrices, $b_{i \alpha}$ are canonical boson operators and a sum over repeated $\alpha$ indices is assumed. Note that we need to impose the constraint $n_{b}=b_{i \alpha}^{\dagger} b_{i \alpha}=2 S$ to satisfy the spin commutation relations, where $S$ is the spin quantum number. A generalized model is obtained by introducing $N$ flavors of such bosons on each site. In order to keep the physical Hilbert space of spins, a constraint on the number of bosons given by $n_{b}=b_{i \alpha}^{\dagger m} b_{i \alpha}^{m}=2 S_{\text {eff }}=\kappa N$ where $m=1, \ldots, N$ must be imposed at each site. Note that $N=1$ corresponds to the physical limit $\mathrm{Sp}(1) \equiv \mathrm{SU}(2)$. The action of the corresponding $\operatorname{Sp}(N)$ generalized model is then given by

$$
\mathcal{S}=\int d \tau\left\{\bar{b}_{i \alpha}^{m} \partial \partial_{i \alpha}^{m}-\frac{J_{i j}}{2 N} \bar{A}_{i j} A_{i j}+\lambda_{i}\left(b_{i \alpha}^{m} b_{i \alpha}^{m}-n_{b}\right)\right\},
$$

where $A_{i j}=\epsilon_{\alpha \beta} \delta_{m m^{\prime}} b_{i \alpha}^{m} b_{j \beta}^{m^{\prime}}\left[\epsilon_{\alpha \beta} \delta_{m m^{\prime}}\right.$ is the $\operatorname{Sp}(N)$ generalized antisymmetric tensor of $\mathrm{SU}(2)]$ and the chemical potential $\lambda_{i}$ keeps the number of bosons fixed to $n_{b}=\kappa N$ at every site. The mean-field action is then obtained by decoupling the quartic boson interaction in $\mathcal{S}$ using the HubbardStratonovich fields $Q_{i j}=-Q_{j i}$ directed along the lattice links so that one obtains $Q_{i j}=\left\langle A_{i j}\right\rangle / N$ at the saddle point. The mean field solution becomes exact in the large- $N$ limit where $N \rightarrow \infty$ is taken while $\kappa=n_{b} / N$ is fixed. We also introduce the parametrization $b_{i \alpha}^{m}=\left(\sqrt{N} x_{i \alpha} b_{i \alpha}^{\widetilde{m}}\right)^{T}$, where $\tilde{m}=2, \ldots, N$ to allow for the possibility of long-range order that occurs when $x_{i \alpha}$ $\neq 0$. Consequently, after integrating over the bosons, we obtain the effective action at the large- $N$ saddle point (or the mean-field free energy) at zero temperature:

$$
\begin{aligned}
\mathcal{S}_{\mathrm{eff}} / N= & \sum_{i, j} \frac{J_{i j}}{2}\left[\left|Q_{i j}\right|^{2}-Q_{i j}\left(\epsilon_{\alpha \beta} x_{i \alpha}^{*} x_{j \beta}^{*}\right)+c . c .\right]+\lambda \sum_{i}\left[\left|x_{i \alpha}\right|^{2}\right. \\
& -(\kappa+1)]+\sum_{\mu} \omega_{\mu}(Q, \lambda)
\end{aligned}
$$

where $\omega_{\mu}(Q, \lambda)$ are the eigenvalues of the mean-field Hamiltonian. Note that the chemical potential is now taken to be uniform since each site has the same number of nearest neighbor links. In general, magnetic ordering $x_{i \alpha} \neq 0$ occurs in the semiclassical limit at larger $\kappa$ while quantum paramagnetic phases are obtained when $\kappa$ is small. In this work, we will study possible phases of such a model as a function of $\kappa$ and $J_{d} / J_{t}$ at zero temperature.

\section{PROJECTIVE SYMMETRIC GROUP ANALYSIS OF $Z_{2}$ SPIN LIQUID PHASES ON THE STAR LATTICE}

We are interested in the classification of Schwinger boson mean-field states, especially the spin liquid phases that do not break any underlying microscopic symmetry. Such symmetric spin liquid phases can be classified using a projective symmetry group analysis, which was previously used for the fermion $^{34}$ and boson ${ }^{22}$ mean-field states for different lattices. For our purpose, the approach taken by Wang and
Vishwanath $^{22}$ would be the most relevant. This analysis allows us to identify all the physically realizable spin liquid phases, independent of particular microscopic Hamiltonians. In this section, we only consider the physical $N=1$ case of the Schwinger boson theory and note that distinct spin liquid phases may be realized as ground states in different models.

In the Schwinger boson theory, the effective action and all physical observables are invariant under the following local $U(1)$ transformation for the boson and mean-field ansatz $Q_{i j}$ :

$$
\begin{gathered}
b_{i \alpha} \rightarrow e^{i \phi(i)} b_{i \alpha}, \\
Q_{i j} \rightarrow e^{-i \phi(i)-i \phi(j)} Q_{i j},
\end{gathered}
$$

where $\phi(i)$ is an arbitrary real field defined on the underlying lattice site. Therefore, two mean-field ansatze that are related by such a transformation correspond to the same physical state after projection (onto the physical Hilbert space). An important point is that symmetry transformations (such as space group, spin rotation, and time reversal) may return a mean-field ansatz to a $U(1)$ transformed form and in this case the transformed ansatz would correspond to the same physical state. Thus when we consider the mean-field ansatz that preserves all the microscopic symmetries, we need to include the $U(1)$ transformations. The main idea of the projective symmetry group analysis is that a mean-field ansatz preserves all the symmetries not only when the ansatz is invariant under the symmetry transformation $X$, but also when it is invariant under the symmetry transformation $X$ followed by a local $U(1)$ gauge transformation, $G_{X}$, i.e.

$$
\left(G_{X} \cdot X\right) Q_{i j}=Q_{i j}
$$

Thus, for example, physically distinct symmetric spin liquid phases can be characterized by different allowed sets of combined transformations, $\left\{G_{X} \cdot X\right\}$.

In addition, there also exist pure local gauge transformations that leave the mean-field ansatz invariant. The set of such elements is called the invariant gauge group (IGG). The IGG is a subgroup of the underlying $U(1)$ symmetry and is not a physical symmetry since it is not related to any microscopic symmetry. On the other hand, the IGG becomes the emergent gauge symmetry in the deconfined phase that describes the relevant spin liquid phases. ${ }^{20}$ Therefore, it is important to identify the IGG of a mean-field ansatz. The IGG and the set $\left\{G_{X} \cdot X\right\}$ together form the PSG. This PSG then can be used to classify the physically distinct spin liquid phases that have the same microscopic symmetries.

It can be readily seen that the IGG of the mean-field ansatz $Q_{i j}$ on the star lattice (or on any frustrated lattice) is $Z_{2}$. The two elements of the IGG are the identity operation $\mathbf{1}$ and the IGG generator $-\mathbf{1}: b_{i \alpha} \rightarrow-b_{i \alpha}$. The spin liquid phases that are characterized by a $Z_{2}$ IGG are called $Z_{2}$ spin liquid states. Here, we would like to classify possible symmetric $Z_{2}$ spin liquid phases on the star lattice using the PSG.

\section{A. Algebraic constraints on the PSG}

We would like to find all the constraints on the PSG that preserve microscopic symmetries such as the space group, spin rotation, and time reversal. The Schwinger boson mean- 
field Hamiltonian is explicitly spin-rotation invariant. Here, we concentrate on the space group operations such as translations and point group operations for the star lattice. The time reversal operation will be considered later. For each space group operation, the allowed gauge transformations in the PSG are strongly constrained by certain algebraic relations among symmetry group elements. Thus, we first need to derive all the algebraic relations (so-called algebraic PSGs) and investigate the solutions which provide all the symmetric spin liquid phases.

In the case of the star lattice, the underlying Bravais lattice is a triangular lattice and the space group contains two translations $T_{1}$ and $T_{2}$ defined by the basis vectors $\mathbf{e}_{1}$ and $\mathbf{e}_{2}$ in Fig. 1, one reflection $\sigma$ along the diagonal, and the $60^{\circ}$ rotation $R$ about a lattice site.

The translation operation, $T_{i}$, shifts the lattice by one unit cell along $\mathbf{e}_{i}$,

$$
\begin{aligned}
& T_{1}:\left(r_{1}, r_{2}, \alpha_{s}\right) \rightarrow\left(r_{1}+1, r_{2}, \alpha_{s}\right), \\
& T_{2}:\left(r_{1}, r_{2}, \alpha_{s}\right) \rightarrow\left(r_{1}, r_{2}+1, \alpha_{s}\right),
\end{aligned}
$$

where $\mathbf{r}=\left(r_{1}, r_{2}, \alpha_{s}\right)$ represents the location of a lattice site. Here $\left(r_{1}, r_{2}\right)$ with integers $r_{1}$ and $r_{2}$ denotes the coordinate of a unit cell $\left(\mathbf{R}=r_{1} \mathbf{e}_{1}+r_{2} \mathbf{e}_{2}\right)$ and $\alpha_{s} \in\{a, b, c, d, e, f\}$ labels the six sites within each unit cell (see Fig. 1). Reflection $\sigma$, however, interchanges the sublattice indices,

$$
\begin{aligned}
& \sigma:\left(r_{1}, r_{2}, a\right) \rightarrow\left(r_{2}, r_{1}, e\right), \\
& \sigma:\left(r_{1}, r_{2}, b\right) \rightarrow\left(r_{2}, r_{1}, f\right), \\
& \sigma:\left(r_{1}, r_{2}, c\right) \rightarrow\left(r_{2}, r_{1}, d\right), \\
& \sigma:\left(r_{1}, r_{2}, d\right) \rightarrow\left(r_{2}, r_{1}, c\right), \\
& \sigma:\left(r_{1}, r_{2}, e\right) \rightarrow\left(r_{2}, r_{1}, a\right), \\
& \sigma:\left(r_{1}, r_{2}, f\right) \rightarrow\left(r_{2}, r_{1}, b\right) .
\end{aligned}
$$

Rotation, $R$, also leaves the 6 sublattice indices interchanged,

$$
\begin{gathered}
R:\left(r_{1}, r_{2}, a\right) \rightarrow\left(r_{1}-r_{2}, r_{1}, d\right), \\
R:\left(r_{1}, r_{2}, b\right) \rightarrow\left(r_{1}-r_{2}, r_{1}, f\right), \\
R:\left(r_{1}, r_{2}, c\right) \rightarrow\left(r_{1}-r_{2}, r_{1}, e\right), \\
R:\left(r_{1}, r_{2}, d\right) \rightarrow\left(r_{1}-r_{2}-1, r_{1}, b\right), \\
R:\left(r_{1}, r_{2}, e\right) \rightarrow\left(r_{1}-r_{2}-1, r_{1}, a\right), \\
R:\left(r_{1}, r_{2}, f\right) \rightarrow\left(r_{1}-r_{2}-1, r_{1}, c\right) .
\end{gathered}
$$

One can define the corresponding gauge transformation $G_{X}$ for each symmetry operation $X=T_{1}, T_{2}, \sigma, R$ :

$$
G_{X}: b_{\mathbf{r} \alpha} \rightarrow e^{i \phi_{X}(\mathbf{r})} b_{\mathbf{r} \alpha} .
$$

The PSG is then generated by combining the $Z_{2}$ IGG and the operations $G_{X} \cdot X$. We follow Ref. 22 for the derivation of the algebraic relations between the PSG elements that would impose strong constraints on possible spin liquid phases and repeat some of the basic arguments here for completeness.

In order to see how the structure of the space group imposes the constraints on the PSG, let us first consider the symmetry operation $T_{1}^{-1} T_{2} T_{1} T_{2}^{-1}$ which is the identity operation:

$$
T_{1}^{-1} T_{2} T_{1} T_{2}^{-1}:\left(r_{1}, r_{2}, \alpha_{s}\right) \rightarrow\left(r_{1}, r_{2}, \alpha_{s}\right),
$$

on every site. It means that the corresponding PSG operations should leave the mean-field ansatz unchanged, namely,

$$
\left(G_{T_{1}} T_{1}\right)^{-1}\left(G_{T_{2}} T_{2}\right)\left(G_{T_{1}} T_{1}\right)\left(G_{T_{2}} T_{2}\right)^{-1} \in \mathrm{IGG} .
$$

The PSG operation above can be rewritten as $\left[T_{1}^{-1}\left(G_{T_{1}}\right)^{-1} T_{1}\right] \cdot\left[T_{1}^{-1} G_{T_{2}} T_{1}\right] \cdot\left[\left(T_{1}^{-1} T_{2} G_{T_{1}}\left(T_{1}^{-1} T_{2}\right)^{-1}\right] \cdot\left(G_{T_{2}}\right)^{-1}\right.$. Since the gauge transformation $Y^{-1} G_{X} Y$ with a space group operation $Y$ acting on a site $\mathbf{r}$ would generate a phase $\phi_{X}(Y(\mathbf{r}))$ in the boson field, the equation above leads to the following constraint

$$
-\phi_{T_{2}}(\mathbf{r})+\phi_{T_{1}}\left[T_{2}^{-1} T_{1}(\mathbf{r})\right]+\phi_{T_{2}}\left[T_{1}(\mathbf{r})\right]-\phi_{T_{1}}\left[T_{1}(\mathbf{r})\right]=p_{1} \pi,
$$

where $p_{1}=0,1$ comes from the fact that there are two elements, $\mathbf{1}$ and $\mathbf{- 1}$ in the IGG.

There are additional constraint equations from other independent space group operations. More specifically, together with Eq. (9), the following symmetry relations need to be taken into account:

$$
\begin{gathered}
T_{2} T_{1}=T_{1} T_{2}, \\
T_{1} \sigma=\sigma T_{2}, \\
\sigma^{2}=1, \\
T_{1} R T_{2}=R, \\
T_{2} R=R T_{1} T_{2}, \\
\sigma R \sigma R=I, \\
R^{6}=1 .
\end{gathered}
$$

It can be shown that all other relations can be derived from them. In the Appendix, we solve all the algebraic constraints derived from these relations. The general solution of the algebraic PSG for the star lattice is found as follows:

$$
\phi_{T_{1}}\left(r_{1}, r_{2}, \alpha_{s}\right)=0,
$$

$$
\phi_{T_{2}}\left(r_{1}, r_{2}, \alpha_{s}\right)=p_{1} \pi r_{1} \text {, }
$$

$$
\phi_{\sigma}\left(r_{1}, r_{2}, \alpha_{s}\right)=p_{1} \pi r_{1} r_{2}+\frac{p_{2} \pi}{2},
$$




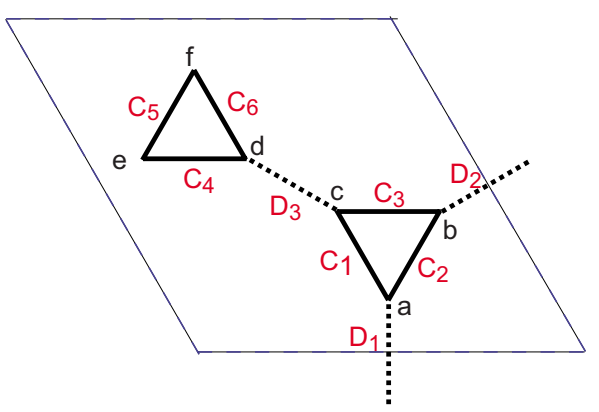

FIG. 2. (Color online) In each unit cell, there are 9 different nearest-neighbor valence-bond amplitude, $Q_{i j}$, which can be classified into two groups, $\left\{C_{1}, C_{2}, C_{3}, C_{4}, C_{5}, C_{6}\right\}$ and $\left\{D_{1}, D_{2}, D_{3}\right\}$.

$$
\phi_{R}\left(r_{1}, r_{2}, \alpha_{s}\right)=p_{1} \pi r_{1} r_{2}+\frac{p_{1} \pi}{2} r_{2}\left(r_{2}-1\right)+\frac{p_{3} \pi}{2}+p_{4} \pi \delta_{\alpha_{s}, f},
$$

where $p_{1}, p_{2}, p_{3}, p_{4} \in\{0,1\}$. Here, $\delta_{\alpha_{s}, f}=1$ when $\alpha_{s}=f$ and zero otherwise. Thus there exist 16 possible symmetric spin liquid phases. Notice that, not surprisingly, the solutions for the translation and reflection are the same as those in the triangular lattice. The solution for the rotation, however, has a more complex structure. The general solution, except for the rotation, looks similar to the one in the triangular and Kagome lattice cases where the underlying Bravais lattice is the same but the number of sites per unit cell is different. However, we will show later that once we consider meanfield ansatz with nonvanishing nearest-neighbor bond amplitudes, $Q_{i j}$, only two of these spin liquid phases survive and the properties of these states are different from the allowed states in the other cases.

To summarize this section, we solve the algebraic PSG constraint equations for the star lattice and find that $p_{1}, p_{2}, p_{3}, p_{4} \in\{0,1\}$ are required to classify all distinct symmetric $Z_{2}$ spin liquid states that preserve all space group symmetries. In the next section, we show that if the nearestneighbor bond amplitudes $Q_{i j}$ are nonzero and time reversal invariance is required, there are additional constraints on these parameters. At a result, we will see that there exist only two symmetric $Z_{2}$ spin liquid states with distinct PSG or quantum order.

\section{B. $Z_{2}$ spin liquid states with nonvanishing nearest-neighbor bond amplitudes}

In the star lattice, there are 9 different nearest-neighbor bond amplitudes $Q_{i j}$ in the unit cell and we label them by $\left\{C_{1}, \ldots, C_{6}\right\}$ and $\left\{D_{1}, D_{2}, D_{3}\right\}$ that correspond to the triangular and bridge links, respectively (see Fig. 2). If we assume that all of them are nonzero, there are more constraints on the PSG structure.

First, we consider what happens to the amplitude $D_{3}(0,0) \equiv Q_{(0,0, c) \rightarrow(0,0, d)}$ (other amplitudes are defined in a similar fashion) under reflection $\sigma$. From Eq. (5c), we infer $D_{3}(0,0) \stackrel{\sigma}{\rightarrow}-D_{3}(0,0)$, then from the definition of the PSG in Eq. (4), we get the constraint $\phi_{\sigma}(0,0, c)+\phi_{\sigma}(0,0, d)=\pi$ $(\bmod 2 \pi)$. This leads to $p_{2}=1$.

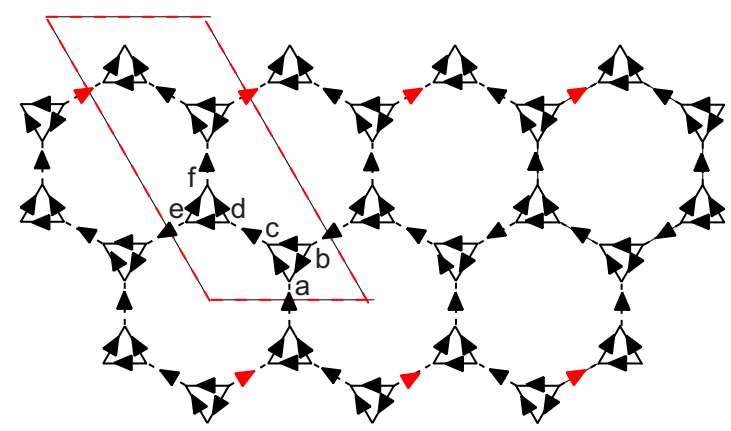

FIG. 3. (Color online) The arrow representation of the meanfield ansatz for the $p_{1}=1$ state. The area enclosed by the dashed lines is the corresponding unit cell with 12 sites. Note that the directed link $Q_{b e}$ is staggered along the $(0,1)$ direction.

Another constraint can be obtained by comparing the $60^{\circ}$ rotation $R$, and the reflection $\sigma$ on $C_{1}(0,0)$,

$$
\left(G_{R} \cdot R\right) C_{1}(0,0)=\left(G_{\sigma} \cdot \sigma\right) C_{1}(0,0) .
$$

Since $C_{1}(0,0) \stackrel{R}{\rightarrow} C_{4}(0,0)$ and $C_{1}(0,0) \stackrel{\sigma}{\rightarrow}-C_{4}(0,0)$, it imposes the condition

$$
\phi_{\sigma}^{d}+\phi_{\sigma}^{e}=\pi+\phi_{R}^{d}+\phi_{R}^{e},
$$

where $\phi_{X}^{\alpha} \equiv \phi_{X}(0,0, \alpha)$ for $X \in\{\sigma, R\}$. This implies that $p_{3}$ $=0$ in the PSG.

Finally, we consider the constraint by $180^{\circ}$ rotation $R^{3}$, and the translation $\left(T_{1}\right)^{-1}$ on the bridge link, $D_{1}(0,0)$,

$$
\begin{gathered}
\left(G_{R} \cdot R\right)^{3} D_{1}(0,0)=\left(G_{T_{1}} T_{1}\right)^{-1} D_{1}(0,0) . \\
R^{3} \\
T_{1}^{-1}
\end{gathered}
$$

Here, $D_{1}(0,0) \rightarrow-D_{1}(-1,0)$ and $D_{1}(0,0) \rightarrow D_{1}(-1,0)$, leading to the constraint,

$$
\begin{aligned}
& \phi_{R}^{c}(0,0)+\phi_{R}^{d}(0,0)+\phi_{R}^{e}(0,0)+\phi_{R}^{b}(-1,0)+\phi_{R}^{a}(-1,0) \\
& +\phi_{R}^{f}(-1,-1)=\pi
\end{aligned}
$$

which implies $p_{4}=1$ in the PSG.

Thus, by assuming nonvanishing nearest-neighbor amplitudes, the parameters which characterize the PSG structure $\left\{p_{1}, p_{2}, p_{3}, p_{4}\right\}$ become $\left\{p_{1}, 1,0,1\right\}$. There are only two distinct symmetric $Z_{2}$ spin liquids corresponding to $p_{1}=0,1$. If the time reversal symmetry is preserved, all the amplitudes $Q_{i j}$ can be taken to be real. Moreover, $Q_{i j}=-Q_{j i}$ that follows from the self-consistent equation. Hence the mean field ansatz $Q_{i j}$ can be depicted by an arrow representation in which the arrow denotes the direction where $Q_{i j}$ is taken to be positive. The arrow representations for the two distinct spin liquid phases are shown in Figs. 3 and 4 respectively. The $p_{1}$ $=1$ state can be described by a unit cell with 12 sites while $p_{1}=0$ state has a unit cell with 6 sites. Both of them are characterized by two kinds of nearest-neighbor bond amplitudes $Q_{d}$ and $Q_{t}$, which refer to the absolute values, $\left|Q_{i j}\right|$, of the amplitudes on the bridge and triangular links, respectively.

These two states can also be distinguished by the "flux" enclosed in a length-12 polygon, ${ }^{27}$ which is defined as the phase $\Theta$ of the gauge-invariant product of the nearest- 


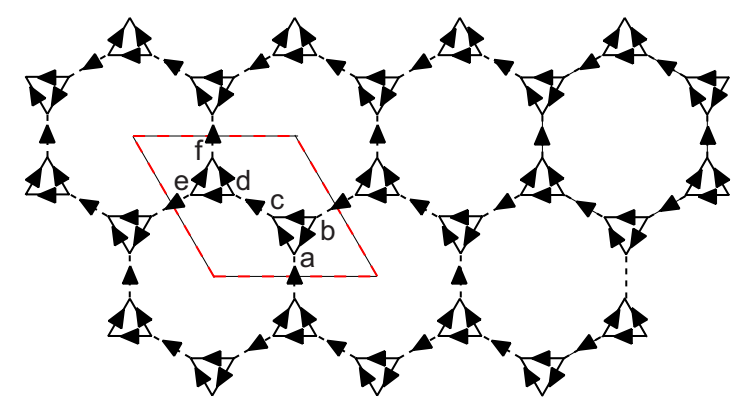

FIG. 4. (Color online) The arrow representation of the meanfield ansatz for the $p_{1}=0$ state. The arrow from the site $i$ to site $j$ means $Q_{i j}>0$. The area enclosed by the dashed lines is the corresponding unit cell with 6 sites.

neighbor amplitudes along the 12-length loop:

$$
Q_{i_{1} i_{2}}\left(-Q_{i_{2} i_{3}}\right) \cdots\left(-Q_{i_{12} i_{1}}\right)=Q_{d}^{6} Q_{t}^{6} e^{i \Theta},
$$

where $\left\{i_{1}, \ldots, i_{12}\right\}$ label the 12 sites along a length-12 loop as shown in Fig. 1. The "flux" $\Theta=0$ for $p_{1}=1$ state (zero-flux state) while $\Theta=\pi$ for $p_{1}=0$ state ( $\pi$-flux state). Hence, the two states are clearly not gauge-equivalent and can be identified by the "flux." The physical properties of these spin liquid states and how they arise in the large- $N \mathrm{Sp}(N)$ meanfield theory will be discussed in the next section.

\section{LARGE- $N$ SP $(N)$ MEAN-FIELD PHASE DIAGRAM}

In this section, we analyze the large- $N$ mean-field theory of the $\mathrm{Sp}(N)$-generalized Heisenberg model with the nearestneighbor exchange interactions. In particular, we investigate the phase diagram as a function of $J_{d} / J_{t}$ and $\kappa=2 S_{\text {eff. In the }}$ previous section, we demonstrate that there are only two possible symmetric spin liquid phases, as shown in Figs. 3 and 4, when the nearest-neighbor bond amplitudes are finite and they correspond to $p_{1}=1,0$ in the PSG description respectively. The strength of the nearest-neighbor bond amplitudes, $Q_{d}$ and $Q_{t}$, and the spinon condensate density $x_{i \alpha}$ can be determined by minimizing the effective action, Eq. (2).

In the $\operatorname{Sp}(N)$-generalized Heisenberg model, it has been known that the spin liquid state with the smallest "flux" has the lowest energy. Thus, not surprisingly, we find that the zero-flux state $\left(p_{1}=1\right)$ is always lower in energy in the relevant part of the phase diagram. On the other hand, it is also known that a ring-exchange or the next-nearest-neighbor spin interactions can lower the energy of a spin liquid state with a larger flux. ${ }^{22}$ Hence, it is useful to analyze the phase diagram of the Heisenberg model with respect to both of the two spin liquid states. The mean-field phase diagram for the nearest-neighbor model is shown in Fig. 5, where the $\pi$-flux state never appears as the true ground state. Anticipating that other types of interactions can favor the $\pi$-flux state, we also compute the mean-field phase diagram by artificially suppressing the zero-flux state (as if an appropriate additional interaction may punish the zero-flux state). The resulting phase diagram is shown in Fig. 6. Notice that the magnetically ordered phases in the large- $\kappa$ limit in Figs. 5 and 6 are descendants of the zero-flux and $\pi$-flux phases in the sense

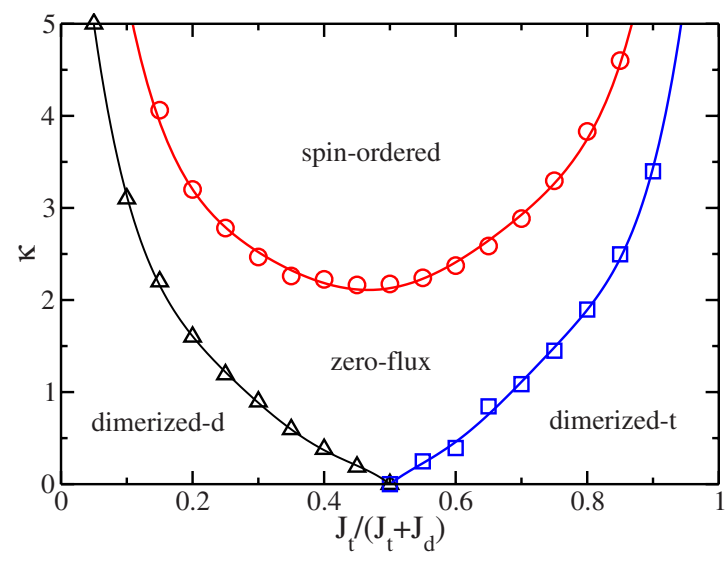

FIG. 5. (Color online) Large- $N$ mean-field phase diagram for the $\operatorname{Sp}(N)$ generalized nearest-neighbor Heisenberg model. Notice that only the zero-flux state occurs as a stable spin liquid state in the phase diagram.

that the condensation of the spinons in each spin liquid state leads to these magnetically ordered phases. On the other hand, the ground states in the $\kappa \ll 1$ limit are typically dimerized or valence bond crystal phases. Physical properties of all the phases present in the phase diagram and the interplay between them are described as follows.

\section{A. zero-flux spin liquid state and the related magnetically ordered phase}

The zero-flux spin liquid state corresponds to $p_{1}=1$ in the PSG description and the mean-field ansatz is shown in Fig. 3. There are 12 sites per unit cell in the mean-field ansatz. It has zero flux in the 12-sided and $\pi$-flux in the 14-sided polygon (see Fig. 1). Hence, it has the lowest energy in the pure Heisenberg model according to the flux expulsion argument by Tchernyshyov et al. $^{27}$ in the small $\kappa$ limit. It is an analogous state of the $[0 \mathrm{Hex}, \pi \mathrm{Rhom}]$ spin liquid state identified in the Kagome lattice. ${ }^{22}$

The spinon spectrum can be computed using the $\operatorname{Sp}(N)$ theory described in Sec. II. However, the single-spinon spec-

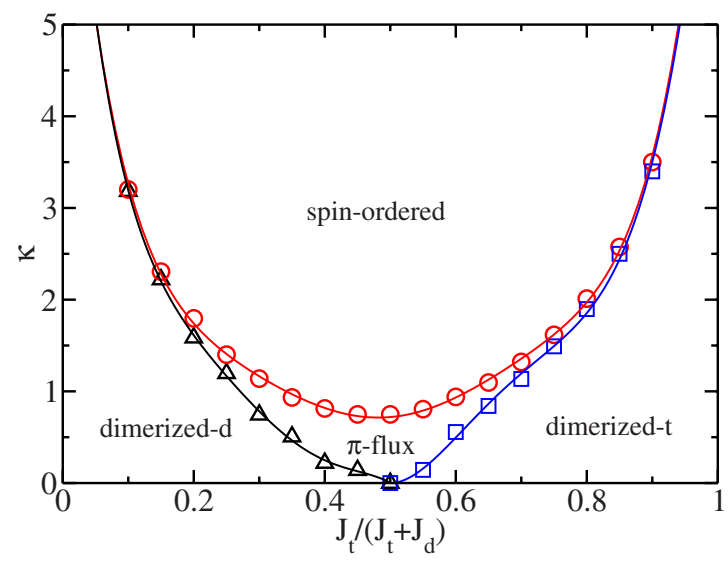

FIG. 6. (Color online) Large- $N$ mean-field phase diagram with the zero-flux state being artificially suppressed (as if an additional interaction punishes the zero-flux state). The $\pi$-flux state is then the spin liquid state competing with the dimerized states. 


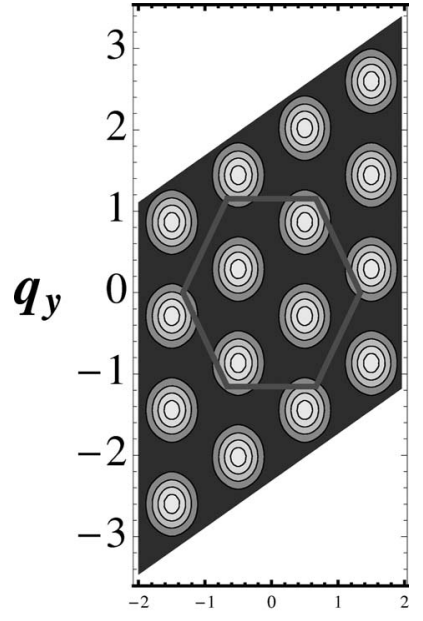

(a)

$q_{x}$

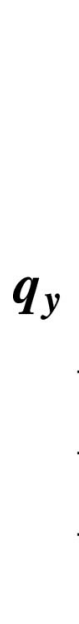

(b)

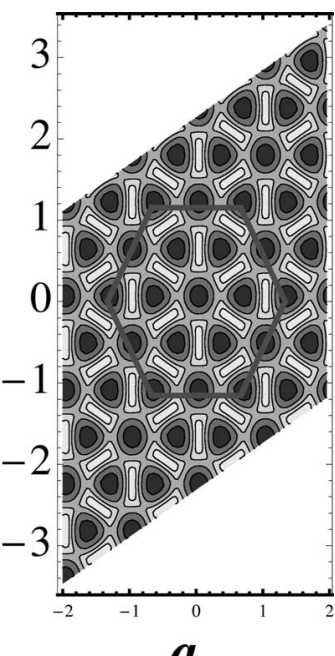

$q_{x}$

FIG. 7. Contour plots of the single-spinon (left) spectrum and the lower edge of the two-spinon/spinon-antispinon (right) spectrum of the zero-flux state $\left(p_{1}=1\right)$. Darker area means lower energy. The hexagon represents the Brillouin zone.

trum is not gauge-invariant and the gauge-invariant twospinon (spinon-antispinon) spectrum is physically more relevant. Here, we present the lower edge of the two-spinon spectrum, which is given by

$$
E^{(2)}(\mathbf{q})=\min _{\mathbf{p}}\left\{\boldsymbol{\epsilon}_{\mathbf{q}-\mathbf{p}}+\epsilon_{\mathbf{p}}\right\}
$$

where $\epsilon_{\mathrm{p}}$ is the single-spinon spectrum. The single-spinon spectrum and the lower edge of the two-spinon spectrum are shown in Fig. 7, which are similar to that of [0Hex, $\pi$ Rhom] spin liquid phase obtained in Kagome lattice. ${ }^{22}$ The minima of two spinon spectrum are given by $\mathbf{q}= \pm(\pi / 3+n \pi, \pi / 3$ $+m \pi)$ and $(n \pi, m \pi)$ with integer $n, m$. As $\kappa$ increases, the minimum of the spinon spectrum decreases and the spectrum becomes gapless at $\kappa=\kappa_{c}$, where $\kappa_{c}=\kappa_{c}\left(J_{d} / J_{t}\right)$ varies depending on the value of $J_{d} / J_{t}$. Possible magnetically ordered phases arising when $\kappa>\kappa_{c}=\kappa_{c}\left(J_{d} / J_{t}\right)$ are characterized by the ordering wavevectors $\mathbf{q}= \pm(\pi / 3+n \pi, \pi / 3+m \pi)$ and $(n \pi, m \pi)$ with integers $n, m$.

\section{B. $\pi$-flux spin liquid state and the related magnetically ordered phase}

The $\pi$-flux spin liquid state is characterized by $p_{1}=0$ in the PSG description and the mean-field ansatz (in the arrow representation) is depicted in Fig. 4. The ansatz is described by a 6 -site unit cell. It has $\pi$ flux in the 12-sided and zero flux in the 14-sided polygon as shown in Fig. 1. Both singleand two-spinon spectrum are shown in Fig. 8. The twospinon spectrum has the global minimum at the center of the Brillouin zone $\mathbf{q}=(0,0)$. It is an analogous state of the $Q_{1}$ $=Q_{2}$ state identified in the Kagome lattice. ${ }^{21,22}$ The condensation of the spinons leads to the $q=0$ magnetically ordered ground state which is translationally invariant. Since the twospinon spectrum of the $\pi$-flux state is quite different from that of the zero-flux state, the two states can be distinguished
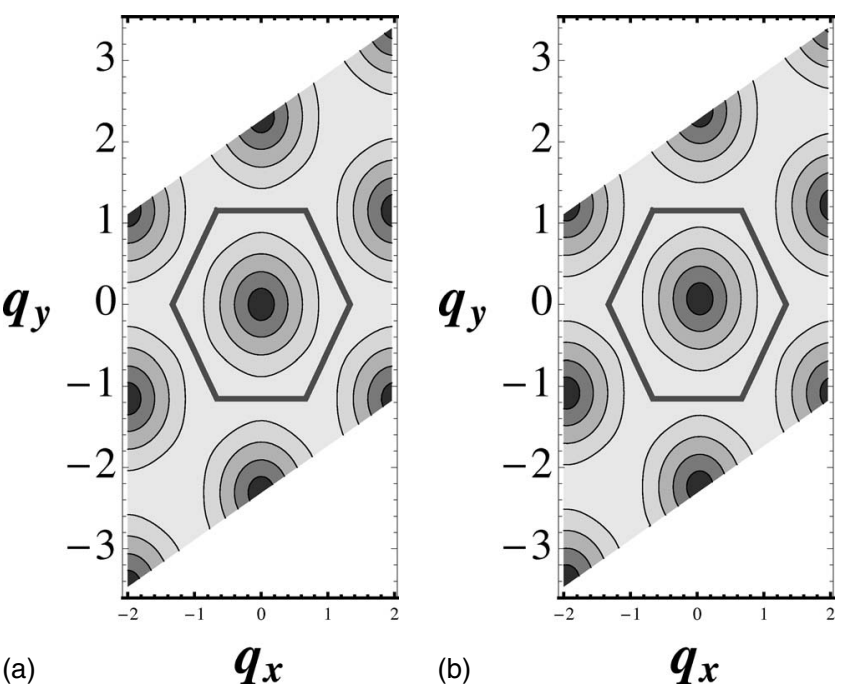

FIG. 8. Contour plots of the single-spinon (left) spectrum and the lower edge of the two-spinon/spinon-antispinon (right) spectrum of the $\pi$-flux state $\left(p_{1}=0\right)$. Darker area means lower energy. The hexagon represents the Brillouin zone.

by neutron scattering experiment that measures spin-1 excitations.

\section{Dimerized $d$ state}

In the regime $J_{t}<J_{d}$, the ground state is a dimerized state for sufficiently small $\kappa$, where all the triangular bond amplitudes vanish $\left(Q_{t}=0\right)$ and only the amplitude on the bridge links, $Q_{d}$, is finite. We call this state as the dimerized-d state. Notice that this state does not break any translational symmetry. This is an isolated-dimer state and there is a gap $\sim J_{d}$ for the spin- 1 excitations. The presence of this ground state in the small $\kappa$ limit can be proven using the small $\kappa$ expansion $^{27}$ of the effective action $S_{\text {eff }}$ in Eq. (2) for paramagnetic solutions $\left(x_{i \alpha}=0\right)$. Such a perturbative expansion of $S_{\text {eff }}$ in $\kappa$ leads to

$$
\frac{S_{\mathrm{eff}}}{N N_{s}}=-\frac{P_{1}}{R} \kappa-\frac{P_{2}}{2 R P_{1}} \kappa^{2}+O\left(\kappa^{3}\right),
$$

where $N_{s}$ is the number of lattice sites, $R \equiv-\left(J_{d} Q_{d}^{2}\right.$ $\left.+2 J_{t} Q_{t}^{2}\right) / 2$, and $P_{n}$ is the "flux operator" defined on the loop of length $2 n$,

$$
P_{n} \equiv \frac{1}{N_{s \text { loop }}} \sum_{2}\left(\frac{J_{12}}{2} Q_{12}\right)\left(-\frac{J_{23}}{2} Q_{23}^{*}\right) \cdots\left(-\frac{J_{2 n, 1}}{2} Q_{2 n, 1}^{*}\right) .
$$

In particular, $P_{1}=-\left(J_{d}^{2} Q_{d}^{2}+2 J_{t}^{2} Q_{t}^{2}\right) / 4$ and $P_{2}=\left(8 J_{d}^{2} Q_{d}^{2} J_{t}^{2} Q_{t}^{2}\right.$ $\left.+6 J_{t}^{4} Q_{t}^{4}+J_{d}^{4} Q_{d}^{4}\right) / 16$.

When $J_{t}<J_{d}$, we find that $Q_{t}=0$ can minimize the effective action Eq. (2) for $\kappa<\kappa_{c}^{d}$ and the critical $\kappa_{c}^{d}$ is

$$
\kappa_{c}^{d}=2 \frac{1-2 u}{4 u-1},
$$

where $u \equiv J_{t} /\left(J_{t}+J_{d}\right) \leq 1 / 2$. This result is asymptotically correct near $u=1 / 2$ where $\kappa_{c}^{d}=0$. When $\kappa>\kappa_{c}^{d}$, the spin liquid 
phases become more stable as far as $\kappa$ is not too large.

\section{Dimerized $t$ state}

When $J_{t}>J_{d}$, there is another dimerized mean-field state for sufficiently small $\kappa$. In this state, all the amplitudes on the bridge links are zero $\left(Q_{d}=0\right)$ while the amplitudes on the triangular links are finite. The mean-field dimerized- $t$ state, therefore, does not break any translational symmetry. Again, the presence of this state can be seen from the small $\kappa$ expansion of the effective action. That is, $Q_{d}=0$ is the solution for the minimum effective action as far as $\kappa<\kappa_{c}^{t}$, where $\kappa_{c}^{t}$ is

$$
\kappa_{c}^{t}=4 \frac{2 u-1}{5-8 u} .
$$

When $\kappa>\kappa_{c}^{t}$, the spin liquid phases become more stable for not-too-large $\kappa$.

\section{E. Further discussions on the phase diagram}

Notice that, at the isotropic point, $J_{t}=J_{d}$, a spin liquid phase becomes the ground state even in the small $\kappa$ limit, where the amplitudes on both the bridge and triangular links are nonvanishing and identical (see Figs. 6 and 5). However, it turns out that the amplitudes on the bridge links become stronger than the ones on the triangular links as $\kappa$ increases. This indicates a tendency to form local singlets on the bridge links, which may be consistent with the results of the exact diagonalization study by Richter et al. ${ }^{23}$ for the spin- $1 / 2$ isotropic model. More precise determination of the ground state at the isotropic point, therefore, requires the analysis of $1 / N$ fluctuations about the large- $N$ mean-field state.

The spin-1/2 anisotropic model with $J_{d} \neq J_{t}$ was previously studied by exact diagonalization restricted to the dimer Hilbert space. ${ }^{24}$ It was found that the dimerized $d$ state is the stable ground state for $J_{t}<1.3 J_{d}$. On the other hand, for the opposite limit $J_{t}>1.3 J_{d}$, it was suggested that the ground state may be a valence bond crystal made of a lattice of 18-sided plaquette-valence-bond structure, which breaks the translational symmetry and is threefold degenerate. ${ }^{24}$ Our mean-field theory cannot capture possible presence of this state since such a state would arise via fluctuations beyond the large- $N$ limit. ${ }^{31,32}$ Thus, the incorporation of relevant quantum fluctuations or another method is necessary to pin down the ultimate fate of the mean-field dimerized $t$ state.

As discussed in the previous sections, the mean-field transition from the spin liquid phases to magnetically ordered phases is continuous since it is described by the condensation of bosonic spinons. When $J_{d}$ and $J_{t}$ are not very different from each other, there is no direct transition from the dimerized state to magnetically ordered phases. On the other hand, in the extreme anisotropic limits, $J_{d} \gg J_{t}$ or $J_{d} \ll J_{t}$, there is a possibility in the $\pi$-flux phase diagram that there is a direct transition from a dimerized state to a magnetically ordered state- the energies of all the states become very close near the phase boundary so that our mean-field calculation could not determine whether there is a direct transition or one still has to go through a spin liquid phase in the extreme anisotropic cases. If a direct transition is possible, such a transition does not have to be always first order because the dimerized $d$ state, for example, does not break any spatial symmetry. The transition from the spin liquid phases to dimerized states (with isolated dimers) is continuous and is described by the confinement-deconfinement transition of spinons in a $Z_{2}$ gauge theory. ${ }^{35}$

\section{DISCUSSION}

In the large- $N$ mean-field phase diagram of the star-lattice Heisenberg model, it is found that the two possible $Z_{2}$ spin liquid phases can exist even for $\kappa>1$ (this corresponds to $S>1 / 2$ in the physical $N=1$ limit) in some parts of the phase diagram. This is highly unusual given that most of the previous studies on other lattice models obtain $\kappa_{c}<1$. While the phase boundaries in the large- $N$ mean-field theory may change as $N$ gets smaller, this is certainly an encouraging sign. Notice, for example, that, when $\kappa=3\left(S_{\text {eff }}=3 / 2\right)$, as $J_{t} /\left(J_{d}+J_{t}\right)$ changes from zero to one, one encounters the dimerized $d$ state, zero-flux spin liquid, magnetically ordered state, zero-flux state and finally the dimerized $t$ state in the mean-field phase diagram (see Fig. 6).

More generally, the zero-flux phase is stable up to a relatively large $\kappa=2 S_{\text {eff }}: \kappa_{c} \sim 2$ at the uniform point $\left(J_{d}=J_{t}\right)$. It is close to the $\kappa_{c}$ obtained in [0Hex, $\pi \mathrm{Rhom}$ ] spin liquid phase in Kagome lattice, which has the similar two-spinon spectrum. $^{22}$ The $\kappa_{c}$ gets even larger in the anisotropic limit, $J_{t} \gg J_{d}$ or $J_{t} \ll J_{d} \cdot{ }^{36}$ The largest $\kappa_{c}$ we obtain is $\kappa_{c} \sim 5$ in a very anisotropic limit, $J_{d} / J_{t} \sim 9$. On the other hand, in such an anisotropic limit, the region in the phase diagram where the spin liquid state is stable becomes smaller. This suggests that moderately anisotropic exchange interactions may favor the realization of spin liquid phases.

It is also worthwhile to notice that the $\pi$-flux state has a relatively small $\kappa_{c}$, in contrast to a similar study of the Kagome lattice model. ${ }^{22}$ As emphasized in Ref. 22, the spin liquid phases with finite flux may be stabilized by a ring exchange term that arise near a metal-insulator transition where charge fluctuations become important. ${ }^{37}$ Thus the starlattice antiferromagnetic insulator at the verge of becoming a metal may be a good candidate for the realization of the $\pi$-flux spin liquid state.

Finally, as far as we know, the only known realization of the star-lattice antiferromagnet is the polymeric iron(III) acetate $\quad\left[\mathrm{Fe}_{3}\left(\mu_{3}-\mathrm{O}\right)(\mu \text {-OAc })_{6}\left(\mathrm{H}_{2} \mathrm{O}\right)_{3}\right]$ $\times\left[\mathrm{Fe}_{3}\left(\mu_{3}-\mathrm{O}\right)(\mu-\mathrm{OAc})_{7.5}\right]_{2} \cdot 7 \mathrm{H}_{2} \mathrm{O} .{ }^{1}$ The spin of the magnetic $\mathrm{Fe}^{\mathrm{III}}$ ion is $S=5 / 2$ and the Curie-Weiss temperature determined from the high-temperature susceptibility is $\Theta_{\mathrm{CW}}=$ $-581 \mathrm{~K}$. This material undergoes a magnetic transition at $T_{N} \sim 4.5 \mathrm{~K}$, leading to a large frustration parameter, $f$ $=\left|\Theta_{\mathrm{CW}}\right| / T_{N}=129$. The magnetic ordering patterns predicted in the large $\kappa$ limit of the large- $N$ mean-field theory may directly be relevant to the low-temperature phase of this system. Once the magnetic ordering pattern is determined by neutron scattering experiment or other means, one may be able to determine whether the material is close to the zeroflux or $\pi$-flux spin liquid phases because they are related to different magnetically ordered phases. ${ }^{28}$ The large frustration parameter observed in this material and the large $\kappa_{c}$ from our 
mean-field theory point to the possibility that a spin-1/2 or even a spin-1 analog of this material may support one of the spin liquid phases discussed in this work.

\section{ACKNOWLEDGMENTS}

This work was supported by the NSERC of Canada, the Canadian Institute for Advanced Research, and the Canada Research Chair Program.

\section{APPENDIX: ALGEBRAIC PSG FOR THE STAR LATTICE}

Here we generalize the method developed in the Ref. 22 to derive the allowed PSGs for the star lattice. The strategy is to find all the constraints on the PSGs and use them to identify the general solution. We first consider how PSG transforms under an arbitrary $U(1)$ gauge transformation $G$ $\equiv e^{i \phi_{G}}$ on the ansatz, $Q_{i j} \rightarrow G Q_{i j}$. The transformed ansatz should now be invariant under $G G_{X} X G^{-1}=\left(G G_{X} X G^{-1} X^{-1}\right) X$. Thus $G_{X}$ can be replaced by $G G_{X} X G^{-1} X^{-1}$. This means that the phase transforms as

$$
\phi_{X}(\mathbf{r}) \rightarrow \phi_{G}(\mathbf{r})+\phi_{X}(\mathbf{r})-\phi_{G}\left[X^{-1}(\mathbf{r})\right] .
$$

Here, $\mathbf{r}=\left(r_{1}, r_{2}, \alpha_{s}\right)$ with integers $r_{1}$ and $r_{2}$ which label the location of the unit cell, $\mathbf{R}=r_{1} \mathbf{e}_{1}+r_{2} \mathbf{e}_{2}$, and $\alpha_{s}$ $\in\{a, b, c, d, e, f\}$ label the six sites in a unit cell.

To simplify the expressions of the PSG, one can choose $\phi_{T_{1}}\left(r_{1}, r_{2}, \alpha_{s}\right)=0$ and $\phi_{T_{2}}\left(0, r_{2}, \alpha_{s}\right)=0$ (independent of the sublattice index $\alpha_{s}$ ), by using a gauge degree of freedom or the gauge transformation $G_{0}$ via

$$
\phi_{G_{0}}\left(r_{1}, r_{2}\right)=-\sum_{i=-\infty}^{r_{1}} \phi_{T_{1}}^{0}\left(i, r_{2}\right)-\sum_{j=-\infty}^{r_{2}} \phi_{T_{2}}^{0}(0, j)
$$

on all sublattices $\alpha_{s}$. Here, $\phi_{T_{1}}^{0}$ and $\phi_{T_{2}}^{0}$ correspond to the phases for an arbitrary initial choice for $G_{T_{1}}$ and $G_{T_{2}}$. Notice that the gauge transformation $G_{0}$ is well-defined only on the lattice with open boundary condition. Extra care is necessary for periodic boundary condition. We assume open boundary condition throughout the analysis for simplicity.

Now we would like to find the PSGs which satisfy all the algebraic constraints in Eq. (11). First, we consider the constraint arising from the symmetry relation, $T_{1} T_{2}=T_{2} T_{1}$, in Eq. (11),

$$
\Delta_{1} \phi_{T_{2}}(\mathbf{r})=p_{1} \pi,
$$

where we introduce two forward difference operators $\Delta_{1}$ and $\Delta_{2}$, defined as $\Delta_{1} f\left(r_{1}, r_{2}\right) \equiv f\left(r_{1}+1, r_{2}\right)-f\left(r_{1}, r_{2}\right)$ and $\Delta_{2} f\left(r_{1}, r_{2}\right) \equiv f\left(r_{1}, r_{2}+1\right)-f\left(r_{1}, r_{2}\right)$. Here $p_{1}=0,1$ is a siteindependent integer corresponding to the two elements in IGG. The solution for $\phi_{T_{2}}$ then is given by

$$
\phi_{T_{2}}\left(r_{1}, r_{2}, \alpha_{s}\right)=p_{1} \pi r_{1},
$$

which is independent of $\alpha_{s}$.

Next, we consider the relation, $\sigma T_{2}=T_{1} \sigma$ and $\sigma T_{1}=T_{2} \sigma$. The constraints arising from these relations are

$$
\Delta_{1} \phi_{\sigma}\left(r_{1}, r_{2}, \alpha_{s}\right)=p_{2}^{\prime} \pi+p_{1} \pi r_{2},
$$

$$
\Delta_{2} \phi_{\sigma}\left(r_{1}, r_{2}, \alpha_{s}\right)=p_{3}^{\prime} \pi+p_{1} \pi r_{1},
$$

after substituting Eq. (A4) for $\phi_{T_{2}}(\mathbf{r})$ and $p_{2}^{\prime}, p_{3}^{\prime}=0,1$. The solution to these equations is

$$
\phi_{\sigma}\left(r_{1}, r_{2}, \alpha_{s}\right)=\phi_{\sigma}^{\alpha_{s}}+p_{2}^{\prime} \pi r_{1}+p_{3}^{\prime} \pi r_{2}+p_{1} \pi r_{1} r_{2},
$$

where $\phi_{\sigma}^{\alpha_{s}} \equiv \phi_{\sigma}\left(0,0, \alpha_{s}\right)$. Here, $p_{2}^{\prime}, p_{3}^{\prime}$ and $\phi_{\sigma}^{\alpha_{s}}$ can further be determined by additional symmetry relations.

Notice that, from $\sigma \sigma=I$, we have

$$
\begin{aligned}
& \phi_{\sigma}\left(r_{1}, r_{2}, a\right)+\phi_{\sigma}\left(r_{2}, r_{1}, e\right)=p_{2} \pi, \\
& \phi_{\sigma}\left(r_{1}, r_{2}, b\right)+\phi_{\sigma}\left(r_{2}, r_{1}, f\right)=p_{2} \pi, \\
& \phi_{\sigma}\left(r_{1}, r_{2}, c\right)+\phi_{\sigma}\left(r_{2}, r_{1}, d\right)=p_{2} \pi .
\end{aligned}
$$

Again $p_{2}=0,1$ correspond to the two elements of the IGG, which is sublattice-independent. Using Eq. (A6), we get the following constraint equation.

$$
\phi_{\sigma}^{a}+\phi_{\sigma}^{e}=p_{2} \pi+\left(r_{1}+r_{2}\right)\left(p_{2}^{\prime}+p_{3}^{\prime}\right) \pi,
$$

and hence, $p_{2}^{\prime}=p_{3}^{\prime}$ (modulo 2) because the left-hand side is independent of $r_{1}, r_{2}$. To determine $p_{2}^{\prime}$, consider the gauge transformation $G_{1}$,

$$
G_{1}: \phi_{G_{1}}\left(r_{1}, r_{2}, \alpha_{s}\right)=\pi r_{1} .
$$

One can show that the gauge transformation $G_{1}$ does not modify $G_{T_{1}}$ and $G_{T_{2}}$, but $G_{\sigma}$ changes as follows:

$$
\phi_{\sigma}(\mathbf{r}) \rightarrow \phi_{\sigma}(\mathbf{r})=\phi_{\sigma}^{\alpha_{s}}+\left(p_{2}^{\prime}+1\right) \pi\left(r_{1}+r_{2}\right)+p_{1} \pi r_{1} r_{2} .
$$

Therefore we can always assume $p_{2}^{\prime}=p_{3}^{\prime}=0$ (modulo 2) and this leads to

$$
\phi_{\sigma}\left(r_{1}, r_{2}, \alpha_{s}\right)=\phi_{\sigma}^{\alpha_{s}}+p_{1} \pi r_{1} r_{2} .
$$

To determine $\phi_{\sigma}^{\alpha_{s}}$, we consider the following gauge transformation,

$$
G_{2}:\left\{\begin{array}{c}
\phi_{2}\left(r_{1}, r_{2}, a\right)=\phi_{0}, \\
\phi_{2}\left(r_{1}, r_{2}, e\right)=-\phi_{0}, \\
\phi_{2}\left(r_{1}, r_{2}, \alpha_{s}\right)=0 \quad \alpha_{s} \notin\{a, e\},
\end{array}\right.
$$

where $\phi_{0}$ is an arbitrary constant. Again, this transformation does not change $G_{T_{1}}$ and $G_{T_{2}}$, but modifies $G_{\sigma}(a)\left(G_{\sigma}\right.$ acting on the sublattice site $a)$ and $G_{\sigma}(e)$ as follows:

$$
\begin{gathered}
\phi_{\sigma}^{a} \rightarrow \phi_{\sigma}^{a}+2 \phi_{0}, \\
\phi_{\sigma}^{e} \rightarrow \phi_{\sigma}^{e}-2 \phi_{0}, \\
\phi_{\sigma}^{\alpha} \rightarrow \phi_{\sigma}^{\alpha_{s}} \quad \alpha_{s} \notin\{a, e\} .
\end{gathered}
$$

By choosing $\phi_{0}=\frac{1}{4}\left(\phi_{\sigma}^{e}-\phi_{\sigma}^{a}\right)$, we can make the phases $\phi_{\sigma}^{a}$ and $\phi_{\sigma}^{e}$ to be equal, i.e., $\phi_{\sigma}^{a}=\phi_{\sigma}^{e}=p_{2} \pi / 2$. Similar gauge transformations, $G_{2}^{\prime}$ and $G_{2}^{\prime \prime}$, can be used for $\{b, f\}$ and $\{c, d\}$ pairs such that all the phases, $\phi_{\sigma}^{\alpha_{s}}$, are chosen to be $p_{2} \pi / 2$. The resulting PSG for the reflection, $G_{\sigma}$, is then given by

$$
\phi_{\sigma}\left(r_{1}, r_{2}, \alpha_{s}\right)=p_{1} \pi r_{1} r_{2}+\frac{p_{2} \pi}{2},
$$

which is independent of the sublattice index, $\alpha_{s}$. 
Now let us consider algebraic constraints arising from $T_{1} R T_{2}=R$ and $R T_{1} T_{2}=T_{2} R$ :

$$
\begin{gathered}
\Delta_{1} \phi_{R}\left(r_{1}, r_{2}, \alpha_{s}\right)=p_{1} \pi r_{2}+p_{4}^{\prime} \pi, \\
\Delta_{2} \phi_{R}\left(r_{1}, r_{2}, \alpha_{s}\right)=p_{1} \pi\left(r_{1}-r_{2}-1\right)+p_{4} \pi,
\end{gathered}
$$

using the solution of $\phi_{T_{1}}$ and $\phi_{T_{2}}$. Here, $p_{4}^{\prime}, p_{4}=0,1$ can be fixed by using additional algebraic constraints and the gauge degrees of freedom. The general solution of the difference equations for $\phi_{R}(\mathbf{r})$ is found as

$$
\begin{aligned}
\phi_{R}\left(r_{1}, r_{2}, \alpha_{s}\right)= & p_{1} \pi r_{1} r_{2}+p_{4}^{\prime} \pi r_{1}+p_{4} \pi r_{2}+\frac{p_{1} \pi}{2} r_{2}\left(r_{2}-1\right) \\
& +\phi_{R}^{\alpha_{s}},
\end{aligned}
$$

where $\phi_{R}^{\alpha_{s}} \equiv \phi_{R}\left(0,0, \alpha_{s}\right)$. To determine $p_{4}^{\prime}$, we consider the relation $R \sigma R \sigma=I$ with Eq. (A15) and Eq. (A17), which results in, for example,

$$
2 \phi_{R}^{a}+p_{4}^{\prime} \pi\left(r_{2}-1\right)=p_{3} \pi
$$

for $p_{3}=0,1$ and hence it implies $p_{4}^{\prime}=0$ (modulo 2). Similarly, we can obtain the following set of coupled equations,

$$
\begin{gathered}
2 \phi_{R}^{a}=p_{3} \pi, \\
\phi_{R}^{b}+\phi_{R}^{c}=p_{3} \pi, \\
\phi_{R}^{d}+\phi_{R}^{e}=p_{3} \pi, \\
2 \phi_{R}^{f}=p_{3} \pi .
\end{gathered}
$$

Unlike the case of the Kagome lattice, there is no further constraint imposed by the relation $\sigma R \sigma R=I$. To fix the gauge degree of freedom for $\phi_{R}^{\alpha_{s}}$, we consider another gauge transformation,

$$
G_{4}=\left\{\begin{array}{l}
\phi_{4}\left(r_{1}, r_{2}, a\right)=\phi_{1}, \\
\phi_{4}\left(r_{1}, r_{2}, e\right)=\phi_{1}, \\
\phi_{4}\left(r_{1}, r_{2}, b\right)=\phi_{2}, \\
\phi_{4}\left(r_{1}, r_{2}, f\right)=\phi_{2}, \\
\phi_{4}\left(r_{1}, r_{2}, \alpha_{s}\right)=0 \quad \text { otherwise. }
\end{array}\right.
$$

This gauge transformation does not modify $G_{T_{1}}, G_{T_{2}}$, and $G_{\sigma}$, but changes $\phi_{R}$ :

$$
\begin{gathered}
\phi_{R}^{b} \rightarrow \phi_{R}^{b}+\phi_{2}, \\
\phi_{R}^{c} \rightarrow \phi_{R}^{c}-\phi_{2},
\end{gathered}
$$

$$
\phi_{R}^{d} \rightarrow \phi_{R}^{d}-\phi_{1}
$$

$$
\phi_{R}^{e} \rightarrow \phi_{R}^{e}+\phi_{1}
$$

$$
\phi_{R}^{\alpha_{s}} \rightarrow \phi_{R}^{\alpha_{s}} \quad \text { otherwise. }
$$

One can show that, by suitable choices of $\phi_{1}$ and $\phi_{2}$, all $\phi_{R}^{\alpha_{s}}$ can be made to be identical and equal to $p_{3} \pi / 2$. To simplify the terms that involve $p_{4}$, we consider another gauge transformation,

$$
G_{5}=\left\{\begin{aligned}
\phi_{5}\left(r_{1}, r_{2}, a\right) & =\pi\left(r_{1}+r_{2}\right), \\
\phi_{5}\left(r_{1}, r_{2}, e\right) & =\pi\left(r_{1}+r_{2}\right), \\
\phi_{5}\left(r_{1}, r_{2}, \alpha_{s}\right) & =\pi\left(r_{1}+r_{2}+1\right) \quad \alpha_{s} \notin\{a, e\},
\end{aligned}\right.
$$

which does not modify $G_{T_{1}}, G_{T_{2}}$ and $G_{\sigma}$, but transforms $\phi_{R}(\mathbf{r})$ as

$$
\begin{gathered}
\phi_{R}\left(r_{1}, r_{2}, \alpha_{s}\right) \rightarrow \\
p_{1} \pi r_{1} r_{2}+\left(p_{4}+1\right) \pi r_{2}+\frac{p_{1} \pi}{2} r_{2}\left(r_{2}-1\right)+\frac{p_{3} \pi}{2} \\
+\pi+\pi \delta_{\alpha_{s} f},
\end{gathered}
$$

Here, $\delta_{\alpha_{s} f}=1$ when $\alpha_{s}=f$ and zero otherwise. In contrast to the case of Kagome lattice where the term $p_{4} \pi r_{2}$ can be gauged away by the transformation $G_{5}$, it becomes $p_{4} \pi \delta_{\alpha_{s}, f}$ in the star lattice. Moreover, we can neglect the constant $\pi$ because it correspond to an IGG operation. Finally, we arrive at

$$
\phi_{R}\left(r_{1}, r_{2}, \alpha_{s}\right)=p_{1} \pi r_{1} r_{2}+\frac{p_{1} \pi}{2} r_{2}\left(r_{2}-1\right)
$$

$$
+\frac{p_{3} \pi}{2}+p_{4} \pi \delta_{\alpha_{s}, f}
$$

${ }^{1}$ Y. Z. Zheng, M. L. Tong, W. Xue, W. X. Zhang, X. M. Chen, F. Grandjean, and G. J. Long, Angew. Chem. Int. Ed. 46, 6076 (2007).

${ }^{2}$ Y. Shimizu, K. Miyagawa, K. Kanoda, M. Maesato, and G. Saito, Phys. Rev. Lett. 91, 107001 (2003).

${ }^{3}$ J. S. Helton, K. Matan, M. P. Shores, E. A. Nytko, Y. Yoshida, Y.
Takano, A. Suslov, Y. Qui, J.-H. Chung, D. G. Nocera, and Y. S. Lee, Phys. Rev. Lett. 98, 107204 (2007).

${ }^{4}$ Z. Hiroi, M. Hanawa, N. Kobayashi, M. Nohara, and H. Takagi, J. Phys. Soc. Jpn. 70, 3377 (2001).

${ }^{5}$ Y. Okamoto, H. Yoshida, and Z. Hiroi, J. Phys. Soc. Jpn. 78, 033701 (2009). 
${ }^{6}$ Y. Okamoto, M. Nohara, H. Aruga-Katori, and H. Takagi, Phys. Rev. Lett. 99, 137207 (2007).

${ }^{7}$ Y. Ran, M. Hermele, P. A. Lee, and X. G. Wen, Phys. Rev. Lett. 98, 117205 (2007).

${ }^{8}$ S. Ryu, O. I. Motrunich, J. Alicea, and M. P. A. Fisher, Phys. Rev. B 75, 184406 (2007).

${ }^{9}$ M. J. Lawler, A. Paramekanti, Y. B. Kim, and L. Balents, Phys. Rev. Lett. 101, 197202 (2008).

${ }^{10}$ Y. Zhou, P. A. Lee, T. K. Ng, and F. C. Zhang, Phys. Rev. Lett. 101, 197201 (2008).

${ }^{11}$ D. Podolsky, A. Paramekanti, Y. B. Kim, and T. Senthil, Phys. Rev. Lett. 102, 186401 (2009).

${ }^{12}$ P. Lecheminant, B. Bernu, C. Lhuillier, L. Pierre, and P. Sindzingre, Phys. Rev. B 56, 2521 (1997).

${ }^{13}$ Ch. Waldtmann, H. U. Everts, B. Bernu, C. Lhuillier, P. Sindzingre, P. Lecheminant, and L. Pierre, Eur. Phys. J. B 2, 501 (1998).

${ }^{14}$ P. Nikolic and T. Senthil, Phys. Rev. B 68, 214415 (2003).

${ }^{15}$ R. R. P. Singh and D. A. Huse, Phys. Rev. B 76, 180407(R) (2007).

${ }^{16}$ B. J. Yang, Y. B. Kim, J. Yu, and K. Park, Phys. Rev. B 77, 224424 (2008).

${ }^{17}$ M. J. Lawler, L. Fritz, Y. B. Kim, and S. Sachdev, Phys. Rev. Lett. 100, 187201 (2008).

${ }^{18}$ F. Wang, A. Vishwanath, and Y. B. Kim, Phys. Rev. B 76, 094421 (2007).

${ }^{19}$ J. Richter, J. Schulenburg, and A. Honecker, in Quantum Magnetism, edited by U. Schollwöck, J. Richter, D. J. J. Farnell, and
R. F. Bishop, Lecture Notes in Physics, Vol. 645 (Springer, Berlin, 2004), pp. 85-153.

${ }^{20}$ X. G. Wen, Phys. Rev. B 65, 165113 (2002).

${ }^{21}$ S. Sachdev, Phys. Rev. B 45, 12377 (1992).

${ }^{22}$ F. Wang and A. Vishwanath, Phys. Rev. B 74, 174423 (2006).

${ }^{23}$ J. Richter, J. Schulenburg, A. Honecker, and D. Schmalfuß, Phys. Rev. B 70, 174454 (2004).

${ }^{24}$ G. Misguich and P. Sindzingre, J. Phys.: Condens. Matter 19, 145202 (2007).

${ }^{25}$ J. O. Fjaerestad, arXiv:0811.3789 (unpublished).

${ }^{26}$ H. Yao and S. A. Kivelson, Phys. Rev. Lett. 99, 247203 (2007).

${ }^{27}$ O. Tchernyshyov, R. Moessner, and S. L. Sondhi, Europhys. Lett. 73, 278 (2006).

${ }^{28}$ S. V. Isakov, T. Senthil, and Y. B. Kim, Phys. Rev. B 72, 174417 (2005).

${ }^{29}$ N. Read and S. Sachdev, Nucl. Phys. B 316, 609 (1989).

${ }^{30}$ J. B. Marston and I. Affleck, Phys. Rev. B 39, 11538 (1989).

${ }^{31}$ J. B. Marston and C. Zeng, J. Appl. Phys. 69, 5962 (1991).

${ }^{32}$ N. Read and S. Sachdev, Phys. Rev. B 42, 4568 (1990).

${ }^{33}$ An alternative $\operatorname{Sp}(N)$ formulation is developed in R. Flint, M. Dzero, and P. Coleman, Nat. Phys. 4, 643 (2008). In our work, we follow the formulation in Ref. 21 for simplicity.

${ }^{34}$ X. G. Wen, Phys. Lett. A 300, 175 (2002).

${ }^{35}$ C. H. Chung, J. B. Marston, and S. Sachdev, Phys. Rev. B 64, 134407 (2001).

${ }^{36}$ M. J. Lawler, H. Y. Kee, Y. B. Kim, and A. Vishwanath, Phys. Rev. Lett. 100, 227201 (2008).

${ }^{37}$ O. I. Motrunich, Phys. Rev. B 72, 045105 (2005). 